\title{
Del saber y de la libertad. El argumento de Isaiah Berlin contra el determinismo (I)
}

\author{
JUAN BOSCO DÍAZ-URMENETA MUÑOZ \\ Universidad de Sevilla
}

Para Rosa e Ignacio

"The New Learning was based on the belief that knowledge and only knowledge the liberated human mind-could save usw1,

\begin{abstract}
RESUMEN
La posición de I. Berlin sobre el determinismo fue recibida por muchos como un ataque al alcance de las ciencias humanas. Su argumentación, sin embargo, sugiere nuevas y sugerentes relaciones entre el conocimiento y la libertad, además de una concepción específica de la lectura de la historia. En el presente ensayo, que constituye una primera parte del trabajo, se analiza el argumento de I. Berlin sobre el determinismo, situándolo en las dos discusiones sobre la historia que se desarrollaron tras la II Guerra Mundial.
\end{abstract}

$$
\begin{aligned}
& \text { PAL.ABRAS CL.AVE } \\
& \text { DETERMINISMO-BERLIN }
\end{aligned}
$$

ABSTRACT

I. Berlin's pasition on determinism was received by many authors as an attack on the human sciences. His interpretation, however, suggests novel and stimulating relations between knowledge and freedom, together with a specific philosophy of history. This paper, which constitutes the firsi part of a whole essay, assesses Berlin's argument on determinism, placing it within the two debates on history held after world war II.

\author{
KEYWORDS
}

DETERMINISM-BERLIN

I. I. Berlin 1990, p. 27 . El nuevo saber denota a la llustración.

\author{
E Contrastes. Revista Intendisciplinar de Filosofia. vol. II (1997), pp. $71-86$ ISSN: 1136-4076 \\ Sección de Filosofía, Universidad de Málaga, Facultad de Filosofia y Letras \\ Campus de Teatinos, E-2907i Mallaga (España)
}


LLEVAR LA TENSIÓN ENTRE LA LIBERTAD Y LA RAZÓN a una discusión teórica, hasta cuestionar que el determinismo sea la conceptualización adecuada del acontecer, es poner en aprietos a la consciencia moderna. Por ello, el ensayo de Berlin, «La Inevitabilidad Histórica» ${ }^{2}$, fue polémico en la filosofía anglosajona y por ello merece aún nuestra atención.

El ensayo es resultado de la reflexión de una época que hay que situar, ante todo, en el contexto de dos discusiones teóricas: 1) la validez y el alcance de las proposiciones y de las categorías específicas del conocimiento histórico y 2) si es posible esperar de la historia, como objeto de conocimiento, regularidades 0 , al menos, modelos para la comprensión del acontecer social. Ambos debates remiten a otros tantos problemas típicos del momento: el de la posibilidad de la ciencia unificada y el del alcance de la planificación social.

El planteamiento de Berlin, sin embargo, va más allá, apunta a la relación entre ciencias humanas y libertad. Su pregunta es si las conceptualizaciones con las que la racionalidad moderna aborda la acción humana no dejan de lado el faktum de la libertad, si estas tramas conceptuales, especialmente las causales y otras más o menos patentemente teleológicas, no llegan a limitar de antemano las posibilidades objetivas de acción-suprimiendo relevancias o descalificando preferencias o negando viabilidad a ciertos ámbitos de acción-, si no llegan a servir de coartada al desconcierto de la subjetividad ante el ejercicio de la opción.

Desde este punto de vista, el contenido del trabajo llega hasta la tercera antinomia kantiana, abordándola no de un modo escolástico, sino desde la experiencia concreta del modo de vida de nuestra época. Esta dimensión la vio con claridad S. Hampshire que escribió a propósito del ensayo:

Confundir la creencia en que los seres humanos no deben ser tratados como si fueran objetos naturales, con la creencia de que en realidad no lo son, es una nueva forma de superstición que, como en otro tiempo las supersticiones religiosas, es una obstrucción al progreso 3 .

Volveré sobre los planteamientos de Hampshire; sólo quiero destacar de momento su certera visión del alcance de la posición de Berlin, en la que no ve una declaración de intenciones de tipo libertario, sino un cuestionamiento de la

2 «Historical Inevitability» fue una conferencia impartida por Berlin como Auguste Comte Memorial Trust Lecture y editada por la Oxford University Press. Se reimprimió en diversas colecciones de ensayos y en 1969 la incluyó su autor en Cuatro Ensayos sobre la Libertad, en cuyo prólogo respondió a algunas de las críticas que se hicieron al ensayo. En la bibliogarfía aparece como Berlin 1988.

3 S. Hampshire 1967, p. 291. Enfasis mío. 
comprensión causal de la acción., con lo que -a juicio de Hampshire- Berlin pone en peligro la posibilidad misma de las ciencias humanas y las posibilidades de previsión racional de la acción. Por ello, Hampshire considera que esa noción de libertad reitera un arcaico espiritualismo y parece un sucedáneo de la noción de alma.

Estos incómodos interrogantes que brotan del ensayo de Berlin no deben disimularse. La reducción que hace Von Wright de su contenido a la autopredicción ${ }^{4}$-ámbito en el que sí podría defenderse la oposición que diseña Berlin entre acontecer y opción, que queda así fuera de la predicción objetivaes, además de inexacta ${ }^{5}$, un modo de aguar el vino. La crítica de Hampshire es; sin embargo, ejemplar porque abre un debate en el que se marcan los perfiles de la actualidad que conserva el problema planteado por Berlin.

Para tratar el problema en toda su amplitud, dedicaré esta primera una primera parte del trabajo a situar el ensayo de Berlin en las dos discusiones que sobre la escritura y la virtualidad de la historia para determinar regularidades sociales se mantienen en la época. Una segunda parte del trabajo estudiará las posiciones de Berlin y sus posibles consecuencias para las ciencias humanas.

Es preciso situar este ensayo, escrito en 1954, en la discusión sobre la explicación histórica. Durante la segunda guerra mundial se abre el debate sobre la explicación científica y.sus consecuencias para la predicción y la atribución causal. Lo inician Hempel y Oppenheim. El texto del primero, que ha quedado como referencia básica de la discusión, se publica en 19486, fecha en la que el debate ha empezado a aplicarse ya a la acción humana y a la historia.

El debate está presidido por la propuesta de la Ciencia Unificada. Toda explicación debe contener leyes generales de modo que lo que se haya de explicar sea consecuencia lógica de esas leyes 7 y de proposiciones empíricas que establezcan la ocurrencia de determinados hechos. Se purifica así la idea de causa de residuos ontológicos y/o mecánicos y se la convierte en noción mediada por leyes generales con contenido empírico y vinculada por nexos lógicos.

Los problemas surgen al extender el modelo de explicación a la historia, algo que, sin embargo, debe poder hacerse porque, el modelo, dice Hempel en 1942 , debe convenir «a cualquier rama de la investigación empírica»8. Pero el

4 G.-H. von Wright 1979, p. 191.

5 I. Berlin 1983a, pp. 299-303.

6 «La Lógica de la Explicación» en C.-G. Hempel 1979, pp. 247-293.

7 Ibid., p. 249.

8 Ibid., pp. 242, 243, 248. 
texto histórico presenta resistencia al explanation sketch. Se habla entonces de una debilidad de la explicación histórica debida a tres razones. La primera es el uso por parte del historiador de principios o generalidades de imposible contrastación empírica, tales como misión histórica ${ }^{9}$, destino o sentido históri$\cos ^{10}$. Esos ejemplos se citan. Una segunda razón es que ciertas fórmulas metodológicas y algunos conceptos de la historia incorporan elementos irreductiblemente subjetivos con lo que no es posible determinar un objeto riguroso del conocimiento: los ejemplos más ilustres son las nociones de comprensión y tendencia. El tercer punto débil es el generoso uso que hace la historia de generalidades procedentes de la psicología individual o social y de muy diversas ramas del saber, $\sin$ que en su empleo se precise el alcance extensional de estas generalidades ni los nexos lógicos que las vinculan. La explicación científica, por el contrario, exige especificar las condiciones empíricas en las que se puede aplicar una generalidad -dicho toscamente, por qué un conjunto de hechos comprobados pueden considerarse ser el caso de tal ley mejor que de tal otra- y excluir por procedimientos lógicos la posibilidad de aplicación de otras.

¿Es posible, pese a todo, incorporar el texto histórico a las exigencias de la explicación? Morton White, en 194311, sugiere que no hay una explicación que pueda considerarse específica de la historia: si emplea con amplitud generalidades de otras ciencias, serán éstas quienes sancionen el alcance de la explicación histórica. Hempel no va tan lejos y sólo propone correcciones vinculadas a los tres problemas ya señalados. Cree que hay que prescindir del primer tipo de generalidades; en cuanto a nociones como comprensión o tendencia, piensa que deben resituarse metodológicamente y precisar su alcance epistemológico: a la noción de comprensión sólo debe atribuírsele un papel heurístico 12 y la de tendencia puede aplicarse sólo al estudio de instituciones y con una especificación empírica tal que pierda todo contenido intencional. En cuanto a las generalidades tomadas de otras ciencias, dado que no es posible cumplir las exigencias del explanation sketch $\sin$ quebrar la escritura normal de la historia, deben tomarse como esbozos de explicación y tratar de perfeccionar su calidad empírica y su alcance lógico. Corregido así el texto histórico, podemos mantenerlo tal como lo conocemos, pero sabiendo que cuanto exceda de los límites propuestos sólo tendrá el rango de interpretación: un texto sugerente, sí, pero sin validez objetiva.

9 C.-G. Hempel 1979 , p. 236.

$10 \mathrm{Ibid}$.

$11 \mathrm{M}$. White 1959.

12 El trabajo básico para la recepción del concepto de comprensión por la filosofía analítica es T. Abel 1948. 
Con ello se consagra, más que la unidad de método, la unidad de objeto del saber. Este debe ser objetivado de acuerdo al procedimiento de la ciencia natural, separado claramente de la apreciación subjetiva del observador, para ser especificado empíricamente con exactitud e incluido en generalidades lógicamente trabadas. A éste objetivismo se añade una reducción de la explicación al desarrollo causal: el acontecer histórico debe ser subsumido en leyes generales y explicado en términos de causa.

Popper incluye en el modelo correcciones de interés. Pensaba que él mismo había iniciado la noción de explicación con su crítica a la idea tradicional de causa ${ }^{13}$, a la que separó del principio de regularidad de la naturaleza y del apriorismo kantiano. Convirtió así a la atribución causal en una cuestión lógica: era la consecuencia lógica de la conjunción formada por una ley general y proposiciones empíricas cuyo alcance se determinaba también por procedimientos lógicos. Popper establece, así, respecto a la observación, una distancia reflexiva que gana densidad si se tiene en cuenta que para él una ley general no es una verdad eterna sino una hipótesis y que la objetividad de un estado del mundo debe precisarse en términos de semántica tarskiana ${ }^{14}$. La explicación es un trabajo reflexivo que debe tener en cuenta la peculiaridad del objeto y la situación del investigador cuya relación con el objeto no es abstracta, sino que está mediada por problemas concretos -la situación-problema-. De este modo los objetos del conocimiento logran una mayor especificidad 15 , y Popper se la reconoce a la historia: mientras la ciencia busca sobre todo determinar la validez general de sus leyes, la historia se interesa más por el suceso particular que por las leyes generales -bastante triviales- que utiliza ${ }^{16}$. Además, las generalidades de las ciencias humanas son diferentes pues, al ocuparse de comportamientos humanos, exigen un conocimiento concreto de situaciones; habrá que precisarlas lógicamente ${ }^{17}$, pero teniendo muy en cuenta que en ellas, 1) hay siempre elementos interactivos que escapan a una clasificación sólo extensional y 2) también hay cuestiones de contenido que una aplicación meramente formal de leyes generales las pasaría por alto. Las relevancias de las ciencias humanas implican, pues una hermenéutica mínima que Popper toma del marginalismo de Menger y de la Deutung weberiana. Popper contribuye, pues, al debate con el reconocimiento de la especificidad del objeto de las ciencias humanas y de la historia, y señalando la peculiaridad de su interés cognoscitivo.

Esta contribución queda, sin embargo, restringida por el vigoroso objetivismo que Popper atribuye al conocimiento. En el caso de la lógica de la situación, el

13 K. R. Popper 1985b, epígrafe 12.

14 K. R. Popper 1984, p. 138, n.28.

15 K. R. Popper 1982 b, nota 7 al cáp. 25.

16 K. R. Popper 1982b, pp. 126-7.

17 K. R. Popper 1984, epígrafe 31; K. R. Popper 1982b, cap. 14. 
afán de objetivación le hace preferir los escenarios institucionales a otros más difícilmente precisables. Es cierto que suaviza esta opción al situar el estudio de la institución en marcos tradicionales ${ }^{18}$, con lo que parece insistir en la dimensión subjetiva de los objetos de la historia. Pero al afirmar, inmediatamente, que las tradiciones deben considerarse en contraste con la tradición racional, el objetivismo se restaura y llega a una confianza ilustrada rayana en la ingenuidad. Este desvío final hacia la objetivación racionalmente controlada se advierte también en su crítica del concepto de comprensión. Podría esperarse que Popper, dado el valor que concede a la reflexión, tomara a la comprensión como categoría reflexiva pero prefiere formalizarla como referencias típicas en las que una cultura establece una situación-problema ${ }^{19}$. De este modo muchos elementos subjetivos se pierden o se trivializan 20 . La opción objetivista de Popper termina por conducirle a una noción de objetividad cercana a la de la ciencia. Popper confía más en su noción tarskiana de verdad objetiva ${ }^{21}$ que en el esfuerzo de Menger por profundizar en los aspectos subjetivos de la vida social, porque piensa que el problema no es lograr una percepción cada vez más fina de las relevancias sociales sino conseguir que los asertos de la sociología se puedan «verificar y corregir por medio de experiencias de tipo objetivo» 22 . Al corregir, es decir, falsar esos asertos, avanza el conocimiento y para lograr verdades falsables es decisivo que se presenten en formas conceptuales impecablemente objetivas. Esta seguridad en la dialéctica de la falsabilidad lo lleva a buscar el objetivismo hasta reintegrar con la unidad de método mucho de lo que había liberado al criticar la unidad de objeto del saber ${ }^{23}$. La consecuencia más importante de esta unidad de método es que Popper termina diferenciando, en las ciencias humanas y en la historia, un saber objetivo, homologable al de la ciencia natural por su rigor conceptual y su

18 K. R. Popper 1983, pp. 156-173.

19 K. R. Popper 1982a, pp. 147-179.

20 Es sorprendente que, para desautorizar el alcance de la empatía, afirme que pese a la comprensión que se les supone «ningún historiador del arte podrá ser un Rembrandt... (K. R. Popper 1982a, p.178). La comunicación intersubjetiva, en consecuencia, sólo podrá estar mediada por objetos reales o lógicos.

21 K. R. Popper 1985a, pp. 188 ss. y 134 ss.

22 K. R. Popper 1982b, nota 14 al cap. 14; énfasis de Popper. Apel subraya que el objetivismo de Popper le incapacita para una comprensión de las ciencias sociales (K.-O. Apel 1985, vol. 2, pp. 215-16). Esto mismo parece verse en la valoración que Popper hace de Weber de quien dice que «desgraciadamente» no vio que ela causalidad se halla vinculada a leyes universales» (K. R. Popper 1982b, nota 7 al cap. 25).

23 Lanzar hipótesis sobre comportamientos sociales, llega a decir, es análogo a proponerlas sobre electrones (K. R. Popper 1984, epígrafes 30 y 31); lo decisivo es que en ambos casos hay una familiarización del investigador con su objeto y la posibilidad objetiva de que los enunciados sean falsables, para lo que deben ser determinados lógicamente. 
falsabilidad, y un amplio campo de hipótesis sugerentes pero no falsables que abandona a una historia escrita como interpretación. Campo que puede dar ideas e iluminar el acontecer pero no generar un conocimiento objetivo.

III

Las posiciones de Berlin -en general y en el ensayo que estudiamos- poco tienen en común con las de Hempel, pero se las suele homologar a las de Popper. Sin embargo hay notables diferencias entre ellas. Berlin piensa que Popper minimiza la diferencia de método entre las ciencias humanas y las naturales 24 . Atribuye, además, gran valor a los elementos subjetivos en la historia y la sociedad, $y$ busca moldes conceptuales para tenerlos en cuenta: por ejemplo, la noción de comprensión que Popper rechaza. La asociación de ambos autores por su creencia en el indeterminismo es un tópico. Berlin dice alguna vez que el argumento popperiano sobre la imposibilidad lógica de la autopredicción no llega a impugnar el determinismo ${ }^{25}$ y que sólo muestra los límites del conocimiento. Los caminos de ambos autores no llegan a coincidir.

Mayor distancia aún hay entre Berlin y los defensores ortodoxos de la aplicación del explanation sketch a la historia y sus diferencias no son sólo epistemológicas sino que en ellas hay implicaciones éticas. Un buen ejemplo puede ser la crítica hacha por Nagel al ensayo que estudiamos, que incorporaría más tarde a su Estructura de la Ciencia ${ }^{26}$. Nagel afirma no defender el determinismo sino la explicación causal. El determinismo sería sólo un principio regulador ${ }^{27}$ cuya función es gnoseológica: postula que para cualquier «conjunto de acciones humanas» o "cambios sociales» de interés para el historiador existe «algún sistema que es determinista respecto a esos elementos»28. En ese marco, Nagel distingue un nivel teórico, propio de la investigación causal, y otro práctico, en el que se desarrolla la acción; los comportamientos se esclarecen en el

24 I. Berlin 1988, p. 114, n. 7. Para completar el cuadro de opiniones sobre el objetivismo de Popper, recordemos el texto de Habermas en el que se dice que su racionalismo convierte a Popper en un ilustrado incapaz de comprender la emergencia de otras culturas o intereses (J. Habermas 1987, pp. 308 ss.).

25 I. Berlin, 1983a, pp. 299-300. El argumento de Popper puede encontrarse completo en K. R. Popper 1986 y compendiado en la introducción a K. R. Popper 1984.

26 E. Nagel 1981. Ver los tres últimos capítulos de la obra; las páginas 537-543 son una crítica expresa a Berlin. El texto inicial de Nagel es E. Nagel, 1960.

27 E. Nagel 1981, 543.

28 E. Nagel 1981, p. 534. Compárese este modo de entender el principio regulativo con la que mantiene Berlin (ver epígrafe IX, en la segunda parte del presente trabajo). El marco que propone Nagel quizá se traduzca en sus análisis de textos históricos que pueden verse en $\mathrm{E}$. Nagel 1981, pp. 506-511. Sobre su valoración de la unidad de método véase E. Nagel 1981, pp. 416 ss. 
primero 29 sin que eso implique un debilitamiento práctico de la voluntad en el segundo ${ }^{30}$ prolonga esos niveles en la distinción entre conducta y acción que pueden estudiarse empíricamente mediante categorías específicas, la de causa para el primer caso y la de motivación para el segundo ${ }^{31}$. Esta distinción, piensa, permitiría, si alguna vez se demostrara ser el determinismo cierto, mantener el lenguaje de la libre opción no sólo con un uso residual -como seguimos diciendo sale el solsino conservando significaciones éticas que inspiran la motivación.

Berlin replica señalando que la ciencia es más una práctica inteligente que una visión del mundo. En ese sentido, la predicción es una tarea de la ciencia, lo mismo que la cuantificación, y para mantenerla no hay que exigir la hipótesis determinista ni aún como idea regulativa, del mismo modo que la cuantificación tampoco precisa que todo sea cuantificable. Pero la crítica fundamental se dirige a la distinción entre conducta y acción. El problema no está en si se puede mantener y cómo el vigor de la motivación bajo la hipótesis de la determinación de la conducta -sea tal hipótesis regulativa o esté testada-, sino en que si al fin llega a mostrarse esta determinación causal, la moralidad -tal como la entendía Kantsería aniquilada ${ }^{32}$. No cabe recurrir al testimonio del voluntarismo para mostrar que la motivación se mantiene en un universo que se sabe determinado 33 . El problema está en que el valor ético de la libertad es intrínseco y no utilitario o emotivista ${ }^{34}$. Aquí se muestra el nervio de la argumentación de Berlin, la preocupación que la cruza. Más tarde entraremos en ella. Lo que interesa destacar ahora es la base de su desacuerdo con la unidad de método de las ciencias naturales y humanas. La predicción científica es una característica del interés cognoscitivo de las ciencias; prolongarla a una visión determinista de la acción tiene importantes implicaciones que no es posible ignorar. Estas implicaciones son en primer lugar de tipo metodológico, pero en cuanto suponen una restricción de la acción al reducírsela a objeto, las implicaciones son también ideológicas. Berlin no desautoriza la pretensión de la unidad de método y objeto de la ciencia, pero mantiene que sus consecuencias para la consideración de la acción deben explicitarse. esas implicaciones deben explicitarse.

IV

La unidad de método es, en esta época, un veto que bloquea la posibilidad de que historia y ciencias humanas cuenten con conceptos y categorías acordes

29 E. Nagel 1981, p. 539.

30 E. Nagel 1981, p. 541.

31 E. Nagel 1981, p. 540.

32 I. Berlin 1988, pp. 37 y 17 , nota 9.

33 1. Berlin 1988, p. 17.

34 I. Berlin 1988, pp. 13-14. 
con la apertura de la subjetividad. Por eso la discusión sufre un fértil giro al desplazarse desde el debate de la explicación histórica al análisis de la escritura y del texto históricos. Con él se iluminan nuevas dimensiones de nociones y procedimientos antes descartados. Así, Gardiner retoma la noción de comprensión 35 , la separa de la lectura idealista de Collingwood ${ }^{36}$ y de todo psicologismo ${ }^{37}$, y la define como «imaginative understanding»38: una operación de la fantasía para penetrar las estructuras simbólicas de otras culturas con la que debe contar toda escritura histórica. Expone que la noción de interpretación es más fértil para la inteligencia de la historia que la atribución causal que por sí sola no da cuenta de lo que es significativo en un estado de cosas; aun la noción de Volkgeist, rechazada por Hempel, puede señalar relevancias que escapan a la causalidad objetiva ${ }^{39}$.

A esta indagación del texto histórico -que incorpora elementos de las Geisteswissenschaften-se unen análisis de inspiración pragmática. Dray mantiene que la explicación histórica no se satisface subsumiendo la información bajo leyes causales, a menudo banales, sino reorganizándola bajo hipótesis 40 que sean iluminadoras. Conceptos ricos, aptos para vincular aspectos muy diversos del acontecer, que precipitan en densos significados son más importantes que las leyes que, al fin, suelen sólo buscarse después de constatar la importancia de un evento. La metáfora y la analogía -epistemológicamente controladas-son más fértiles para perfilar lo nuevo, que la ley, de ordinario demasiado general y pálida ${ }^{41}$. Merece la pena observar que algunas de estas apreciaciones se aproximan a la tradición historiográfica alemana. Los «conceptos dominantes» o «ideas maestras» que, según Dray, iluminan los hechos traen ecos de Ranke, aunque su fuente explícita sea Walsh ${ }^{42}$.

La reflexión de Scriven ${ }^{43}$ sobre el texto histórico une aspectos de esas tradiciones. Las propiedades formales que exige la explicación son insuficientes cuando se examinan críticamente desde el interés cognoscitivo propio de la escritura de la historia. Ésta no sólo exige una adecuación formal sino un análisis de contenido y una indagación reflexiva de las categorías que resulten más ajustadas a la natura-

35 P. Gardiner 1961. El texto apareció en su primera edición en 1952.

36 P. Gardiner 1961. Para el concepto de comprensión en Collingwood, en su aspecto más descriptivo, véase R. G. Collingwood 1974; de modo más teórico y en donde es fácil ver las influencias de Croce, R. G. Colligwood 1984.

37 P. Gardiner 1961. Ver su introducción a la parte II de la compilación, p. 268.

38 P. Gardiner 1961, p. 132.

39 P. Gardiner 1961, p. 112; ver nota al pie de esa página.

40 W. Dray 1959, pp. 403-404.

41 W. Dray 1959, pp. 407.

42 W. H. Walsh 1985. El pasaje clásico para la noción de ideas rectoras en Ranke es la introducción a sus conferencias a Maximiliano de Baviera. Hay edición española: Sobre las épocas de la Historia Moderna. Madrid: Editora Nacional, 1984. 
leza del asunto. La relevancia de la explicación, en el caso del texto histórico, tampoco viene dada por una consideración formal del sujeto y el objeto sino por criterios que comparten historiador y lector: sólo en esa relación pueden determinarse las preguntas a las que inexcusablemente hay que responder y los aspectos de una situación que en absoluto pueden pasarse por alto.

Berlin buscaba zonas libres para el acontecer humano. Las que proporciona esta discusión no proceden de la consideración ética de la acción sino simplemente del análisis del texto y la escritura de la historia: del quehacer del historiador. Berlin insistirá, en el ensayo que estudiamos, en esas dimensiones de la tarea del historiador. Lo hará siguiendo las posiciones de Weber en la selección de relevancias. Pero ya en este ensayo se anuncian exigencias más radicales: manteniendo la definición aristotélica de la historia, su preocupación apunta a una escritura de la historia que insista en los aspectos diferenciales de culturas y formas de vida. Para ese tipo de investigación histórica encontrará importantes claves en la obra de Vico y en la de Herder, que relacionará a veces con conceptos diltheyanos. Más tarde entraré en ellos.

De momento baste señalar que el giro que sufre la discusión sobre el alcance de la escritura de la historia, que podríamos calificar como un paso simultáneo hacia la hermeneutica y al pensamiento pragmatista. logra conceptos aptos para la consideración reflexiva de la acción. Aptitud que quizá brote del origen idealista de esas nociones que ahora se han separado de toda pretensión ontológica. En este ámbito se inscribe Berlin aunque su radicalización en la visión individualizada de culturas y formas de vida plantea un nuevo problema: si este tipo de análisis no termina renunciando a una visión general del acontecer $44 \mathrm{y}$, si llega a consideraciones de interés, éstas son tan dispersas que no logran ofrecer una visión de la historia. Berlin responderá a esto y ampliará el espacio abierto por el giro pragmático de la discusión.

V

La época conoce otro gran debate, el que mantienen Popper y Hayek sobre la filosofía de la historia y sus relaciones con la teoría social. Su horizonte es político porque la crítica no apunta sólo a las filosofías de la historia sino a los modelos de planificación social que inspiran.

La discusión se centró en lo que ambos autores llaman historicismo. Historicista es quien desplaza la teoría social a la historia porque cree que en

43 C. Scriven 1959.

44 P. Gardiner 1959, introducción a la segunda parte de su compilación, página, 271. Una discusión ya clásica sobre las dificultades de acceder a la generalidad desde la comprensión puede verse en J. Habermas 1982. 
ésta se manifiestan las fuerzas que operan en el desarrollo social 45 ; la historia permitiría correlacionar estas fuerzas y llegar a leyes generales o a un modelo teórico del acontecer con lo que podría definirse el conjunto de la vida social 46 . Este desplazamiento, creen, viene impulsado además por la convicción de que en la historia es donde puede surgir la novedad a la que la ciencia social sólo llega ex post festum. El historicista convierte a la historia en ámbito de predicción, piensa Popper, o -a juicio de Hayek- en escenario de fenómenos que pueden estudiarse en su génesis y evolución 47 .

Las críticas de ambos autores no son coincidentes. Popper rechaza la posibilidad de unas leyes históricas asimilables a las científicas porque, piensa, la historia no puede precisar las condiciones iniciales de los fenómenos ni el contenido lógico de las hipótesis ${ }^{48}$. La historia por lo tanto no dispone de leyes. Hayek es más radical: insiste en el carácter singular de los fenómenos históricos que son, por eso, difíciles de formalizar: generalizarlos no es sino un confuso intento entre la inducción y la descripción 49 . Ambas críticas son próximas, pero mientras Hayek parte de una óptica cercana a las Ciencias del espíritu, Popper procede desde lo que más arriba hemos llamado unidad de método de la ciencia.

Ambos niegan que las filosofías de la historia puedan determinar con rigor sus conceptos. Para Hayek, los fenómenos sociales son específicos de las diversas épocas y culturas; logramos entenderlos gracias a la comprensión de las categorías de esas sociedades y así hablamos de salario, trabajo o Estado50, pero la determinación no es unívoca y el alcance de tales conceptos deberá precisarse en cada civilización, cultura o época. La debilidad teórica del historicismo está en generalizar indebidamente sus objetos al inscribirlos en una racionalidad abstracta, separada de los procesos reales. Hayek cree en la deriva histórica de la racionalidad pero como un proceso, inseparable del hábitosi y el acuerdo 52 , que es además "orgánico, lento, semiinconsciente» 53 . La crítica de Popper se dirige, más que a la generalización indebida de los conceptos, a la imprecisión de las llamadas leyes históricas. Éstas ocultan su inestable contenido empírico y su incapacidad para determinar situaciones iniciales bajo la distin-

45 K. R. Popper 1984, epígrafe 16; F. A. von Hayek 1954, pp. 112-114.

46 K. R. Popper 1984, epigrafes, 24 y 25.

47 F. A. von Hayek 1954, p. 117.

48 K. R. Popper 1984, epígrafes 27 y 28. Para la idea de contenido empírico y lógico de las hipótesis, ver K. R. Popper 1983, pp. 459-77. De modo menos técnico puede hallarse en K. R. Popper 1985a, epígrafe 7.

49 F. A. von Hayek 1954, pp. 121-125.

50 F. A. von Hayek 1954, p. 152.

51 F. A. von Hayek 1982 a, p. 91.

52 F. A. von Hayek 1982a, p. 93.

53 F. A. von Hayek 1982 a, p. 86. 
ción entre esencia y apariencia ${ }^{54}$. Con ella, los fenómenos históricos remiten a una dimensión más profunda que, para Popper, es siempre indeterminada. Esa estructura del objeto permite al historicismo prescindir de ciertas relevancias empíricas y proponer, sin control crítico, nuevas condiciones iniciales cuando las hipótesis fracasan. La distinción entre esencia y apariencia sería entonces un inmunizador de la crítica ${ }^{55}$. ¿Por qué puede mantenerla el historicismo? Porque mantiene una noción abstracta del conocimiento, separada de la pregunta y del problema, y que conserva la ilusoria pretensión de que el saber es completo 56 . Se reitera así la sospecha sobre los universales del saber histórico pero desde otro punto de vista. Popper, coherentemente con sus planteamientos, no aceptará el recurso a la comprensión y su visión del acontecer no está tan cargada de escepticismo como en el caso de Hayek. Los análisis del acontecer pueden y deben hacerse pero en marcos institucionales y tradicionales que sitúen a la acción en correlatos concretos. Popper cree que es posible un reformismo político pero sus propuestas deben hacerse desde tales tramas institucionales y con un control cuasi-científico de la hipótesis de manera que estas puedan ser evaluadas en su alcance de acuerdo al método de conjetura y refutación.

Se advierten, pues, las diferencias entre ambos autores. La heterogeneidad entre los objetos del saber histórico acercan a Hayek al escepticismo y lo llevan a emplear modos de interpretación que Popper cuestionaría. Éste, por su parte, mantiene una posición intervencionista en la sociedad, lo que supone que es posible captar ciertas líneas del acontecer. A la oscura deriva de la racionalidad que defiende Hayek, Popper puede oponer una idea de progreso institucional57.

Popper, desde luego, negará que pueda atribuirse a la historia un fin o una dirección, menos aún una meta o un sentido. Pero si el conocimiento permite cierta intervención en el acontecer, el comportamiento racional, al ser sobre todo una opción ética 58 , abre una posibilidad de consenso si bien limitada a evitar ciertas carencias de nuestra sociedad -pobreza, discriminación racial, paro, enfermedades- por medio de una tecnología social fragmentaria. El acuerdo posible se centra, pues, en combatir males, es un acuerdo sobre lo negativo, como lo es el conocimiento en Popper. Algo que está también presente en su propuesta de tecnología social fragmentaria que, por ser tal, ha de estar controlada por las alternativas de conjetura y refutación. Estas limitaciones no impiden que en Popper haya una idea autorreflexiva de la historia: es posible una

54 K. R. Popper 1982b, pp. 91-92.

55 K. R. Popper 1983, pp. 375 ss.

56 K. R. Popper 1983, p. 137.

57 Sobre las nociones popperianas «tecnología fragmentaria» y «progreso intitucional», en un plano básico y claro, ver K. R. Popper 1984. Uno de los textos más sugerentes sobre la idea de progreso se encuentra, sin embargo, en K. R. Popper 1982a, pp. 138 ss.

58 K. R. Popper 1982b, cap. 24, epígrafe II. 
intervención consensuada racionalmente sobre el acontecer 59 , que oriente la cooperación interhumana y la comunicación en torno a valores - o contravaloresgenerales.

La posición de Hayek es mucho más escéptica. Un consenso sobre fines, aun negativos, es una ilusión porque el juicio subjetivo y la preferencia individual son los impredictibles protagonistas del acontecer e intentar formalizarlos es engañarse. En los años 70, cuando Hayek inicia una verdadera cruzada contra el keynesianismo, señala que si las leyes del mantenimiento de la demanda son falsables, no son por ello científicas. Son inadecuadas porque no prevén que los agentes sociales manipularán en su beneficio los escenarios que proponen esas leyes y negarán de facto su alcance. Popper confía en una acción moderada por la racionalidad, Hayek piensa que los factores subjetivos son ingobernables y que no cabe más que inscribirlos con firmeza en los dispositivos liberales, el imperio de la Ley y el mercado. Aquél garantiza la iniciativa del individuo, la igualdad interindividual y la previsibilidad de las conductas; éste ofrece la única información fiable sobre preferencias y necesidades ${ }^{60}$. Por lo demás, pone bajo sospecha aún a los organismos de representación democrática $^{61}$ y opta, frente a ellos, por colegios legislativos de notables, cerrando así su ciclo contra los fantasmas de la planificación.

VI

El destino de este debate es paradójico porque parece conducir a limitaciones tanto de la planificación como del liberalismo que proponen. En efecto: el debate es una potente crítica para las lecturas mitológicas de Marx, tan frecuentes en la época, y baña de incertidumbre conceptos tales como fases de desarrollo social o tendencias históricas pero al faltar en ambos autores una comprensión adecuada de la dialéctica y percibir al socialismo con la estrechez de una doctrina, muchas de sus críticas pierden profundidad. Por otra parte, la crítica que hace Hayek de determinados conceptos generales termina por conducirlo a un cuasi-empirismo en el terreno teórico y a postular, en la práctica política, procedimientos antidemocráticos. Popper, por su parte, confía en procedimientos racionales para determinar ciertas formas del acontecer y para fijar formas de consenso, pero su objetivismo hace que las propuestas prácticas no estén exentas de una cierta tecnocracia, mientras que el consenso deba descansar sobre un optimismo ilustrado que no esté libre de ingenuidad. En la columna del haber, no obstante, hay que anotar el carácter reflexivo de

59 Para el concepto de unidad racional del género humano, K. R. Popper 1982b, cap. 10.

60 F. A. von Hayek 1982b, capítulos 9 y 12.

61 F. A. von Hayek 1982b, capítulos 6 y 10. 
los conceptos que pueden determinar el acontecer y de las propuestas que puedan alcanzar el consenso.

Suele asociarse a Berlin con los protagonistas de esta discusión. Su posición, sin embargo, es algo diferente. Comparte el rechazo a las filosofías de la historia con pretensiones de saber completo: son, dice, nuevas ontologías porque sustancializan sus categorías, sólo que tal acusación es difícil hacerla a Marx, cuyo tratamiento de la historia es más empírico que doctrinario. Para Berlin, la obra de Marx tiene las limitaciones de las construcciones racionalistas que creen poder establecer de antemano unos ámbitos relevantes de la acción -en lo que a procedimientos y fines se refiere-frente a otros que no lo son. Berlin piensa que los fines individuales difieren radicalmente e incluso de oponen y que en los procedimientos puede haber similar variedad. Esta crítica a Marx puede dirigirse igualmente a las propuestas popperianas, tanto en lo que a la planificación fragmentaria se refiere como en lo que toca a las posibilidades de consenso. Berlin, en ambos casos, guarda un prudente escepticismo, afirmando sólo que lo decisivo es defender ámbitos de opción individual. Pese a ello, no se entrega a la desnuda lógica de los reguladores de la acción en los que confía Hayek: ni el mercado ni la pureza del procedimiento llegan a garantizar formas de convivencia que no hieran intereses o fines individuales o valores que un grupo establezca como decisivos. Lo verdaderamente importante es reconocer el pluralismo, aun en su capacidad de suscitar conflictos insolubles, y defender desde ahí una vida decorosa para la mayoría, con canales de intercomunicación que eviten la violencia. Las formas de consenso racional quizá no logren garantizar el pluralismo, los mecanismos de mercado no avalan esta intercomunicación en torno a esa unión que propone Berlin entre criterios aristotélicos y utilitarios.

Estas concepciones de Berlin tienen una importante consecuencia: la historia no es desde luego un desarrollo determinable racionalmente, pero tampoco una lenta deriva práctica de la acción racional o un desarrollo lineal de instituciones; la historia es un despliegue de diferentes culturas cuyos puntos de referencias cognoscitivos y valorativos son muy diversos, casi cabría decir inconmensurables. Este despliegue heterogéneo puede ser, sin embargo, comprendido por quien logre penetrar en esos diferentes mundos. Tal comprensión convierte al estudio de la historia en un ejercicio de reflexión que, al esforzarse en captar la diferencia, se convierte en la mejor escuela de pluralismo.

Al situar a Berlin en estas dos discusiones obtenemos valiosos elementos para entrar en el análisis de su ensayo sobre el determinismo. La acción humana está cargada de una incertidumbre que no se rinde a regularidades de tipo científico objetivo; tales regularidades empobrecen el alcance y el significado de la acción. Uno y otro tampoco se esclarecen uno y otro desde conceptos teleológicos. Esta irreductibilidad a conceptos determinados no acaban, sin 
embargo, con la escritura de la historia ni la privan de sentido. Es cierto que el texto histórico no puede considerarse como un encadenamiento de causas a efectos ni puede constituir el escenario de una Razón triunfante. El texto histórico desgrana significados de la acción humana y propone formas integradas de experiencia y no lo hace para una erudición vacía sino para lectores que se preguntan por el sentido de ciertos acontecimientos que juzgan importantes. Situado en este pliegue reflexivo, el texto histórico, más que dar una razón monológica del acontecer, despliega una enorme dispersión de fines y significados de la acción: es una fenomenología de la diferencia.

Esta amplia consideración de la historia nos permitirá entrar con mayor claridad en el argumento de Berlin sobre el determinismo que abordaremos a continuación.

\section{REFERENCIAS BIBLIOGAFICAS}

ABEL, T. 1948: «The Operation called 'Verstehen'», American Journal of Sociology, 53 (1948), pp. 211 ss.

APEL, K.-O. 1985: La Transformación de la Filosofia, tr. A. Cortina, J. Chamorro y J. Conill, Madrid: Taurus.

BERLIN, I. 1983a: Conceptos y Categorías, tr. F. González Aramburo, México: Fondo de Cultura Económica.

1988: Cuatro Ensayos sobre la Libertad, tr. (de la edición de 1969 de Four essays on Liberty) B. Urrutia, J. Bayón y N. Rodz. Salmones, Madrid: Alianza. 1990: The Magus of the North. J. G. Hamann and the Origin of the Modern irrationalism, H. Hardy, ed., London: John Murray.

COLlingwOOD, R. G. 1974: Autobiografía, tr. J. Hernández Campos, México: Fondo Cultura Económica.

1984: Idea de la Historia, tr. E. O’Gorman y J. Hernández Campos, México: Fondo Cultura Económica.

DRAY, W. 1959: «'Explaining What' in History». en P. Gardiner 1959, pp. 403-408.

GARDINER, P. 1959: Theories of History, London: The Free Press. 1961: The Nature of Historical Explanation, Oxford: Oxford University Press.

HABERMAS, J. 1982: Conocimiento e Interés, tr. M. Jiménez, J. F. Ivars y L. Martín Santos, rev, por J. Vidal Beneyto, Madrid: Taurus.

1987: Teoría y Praxis. Estudios de Filosofia Social, tr. S. Mas y C. Moya, rev. por J. Muñoz, Madrid: Tecnos.

HAMPSHIRE, S. 1967: «Philosophy and Madness», Listener, 87 (1967), pp. 289-292.

HAYEK, Fr. A. von 1982a: Los Fundamentos de la Libertad, tr. J. V. Torrente. Buenos Aires: Centro Estudios. 1982b: New Studies in Philosophy, Politics, Economics and the History of Ideas, London: Routledge and Kegan Paul. 1954: Scientism and Social Sciences, New York: Rowlands.

HEMPEL, C. G. 1979: La explicación científica. Estudios sobre la filosofía de la ciencia, tr. M. Frassineti, N. Míguez, I. Ruiz Aused y C. S. Seibert, Buenos Aires: Paidós. 
NAGEL, E. 1981: La Estructura de la Ciencia. Problemas de la Lógica de la Investigación Cientifica, tr. N. Míguez rev. por G. Klimovsky, Barcelona: Paidós Ibérica. 1960: «Determinism in History», Philosophy and Phaenomenological Research, 20 (1960), pp. 311-317.

POPPER, K. R. 1982a: Conocimiento Objetivo, tr. C. Solís Santos, Madrid: Tecnos. 1982b: La Sociedad Abierta y sus Enemigos, tr. C. Loedel, Barcelona: Paidós Ibérica.

1983: Conjeturas y Refutaciones. El desarrollo del Conocimiento Científico, tr. M. Grasa, Barcelona: Paidós.

1984: La Miseria del Historicismo, tr. P. Schwartz, Madrid: Alianza.

1985a: Búsqueda sin término. Una Autobiografía Intelectual, tr. G. Trevijano. Madrid: Tecnos.

1985b: La Lógica de la Investigación Científica, tr. V. Sánchez Zabala, Madrid: Tecnos. 1986: El Universo Abierto. Un Argumento en favor del indeterminismo. Post Scriptum a la Lógica de la Investigación Científica, vol. III, tr. N. Sansigre Vidal. Madrid : Tecnos.

SCRIVEN, C. 1959: «Explanation and Interpretation in History» en P. Gardiner 1959, pp. 408-427. VON WRIGHT, G. H. 1979: Explicación y comprensión, tr. I. Vega Reñón. Madrid: Alianza. WALSH, W. H. 1985: Introducción a la filosofía de la historia, tr. F. Torner. México: Siglo XXI. WHITE, M. 1959: «Historical Explanation» en P. Gardiner 1959, pp. 357-373. 1956: Review on «Historical Inevitability», Perspectives USA, 16 (1956), pp. 19-26.

Juan Bosco Díaz-Urmeneta es profesor asociado de Estética y Teoría de las Artes en la Universidad de Sevilla. Autor de Individuo y racionalidad moderna. Una lectura de Isaiah Berlin (Sevilla: Universidad de Sevilla, 1994), así como de la versión castellana introducción y notas a I. Berlin, El Mago del Norte. J. G. Hamann y los orígenes del irracionalismo moderno (Madrid: Tecnos, 1997).

Dirección Postal: Universidad de Sevilla, Facultad de Ciencias de la Información, Cl. Gonzalo Bilbao, 7-9, Sevilla. 